\section{GREEN TEA AND ORAL HEALTH EXAMINED IN STUDY}

Drinking green tea may help promote healthy teeth and gums according to new research.

A study recently published in the Journal of Periodontology, the official publication of the American Academy of Periodontology (AAP), analysed the periodontal health of 940 men and found that those who regularly drank green tea had superior periodontal health than subjects that consumed less green tea.
With origins dating back over 4,000 years, green tea has long been a popular beverage in Asian culture, and while ancient Chinese and Japanese medicine believed green tea consumption could cure disease and heal wounds, recent scientific studies are beginning to establish the potential health benefits of drinking green tea, especially in weight loss, heart health, and cancer prevention.

'It has been long speculated that green tea possesses a host of health benefits', said study author Dr Yoshihiro Shimazaki of Kyushu University in Fukuoka,

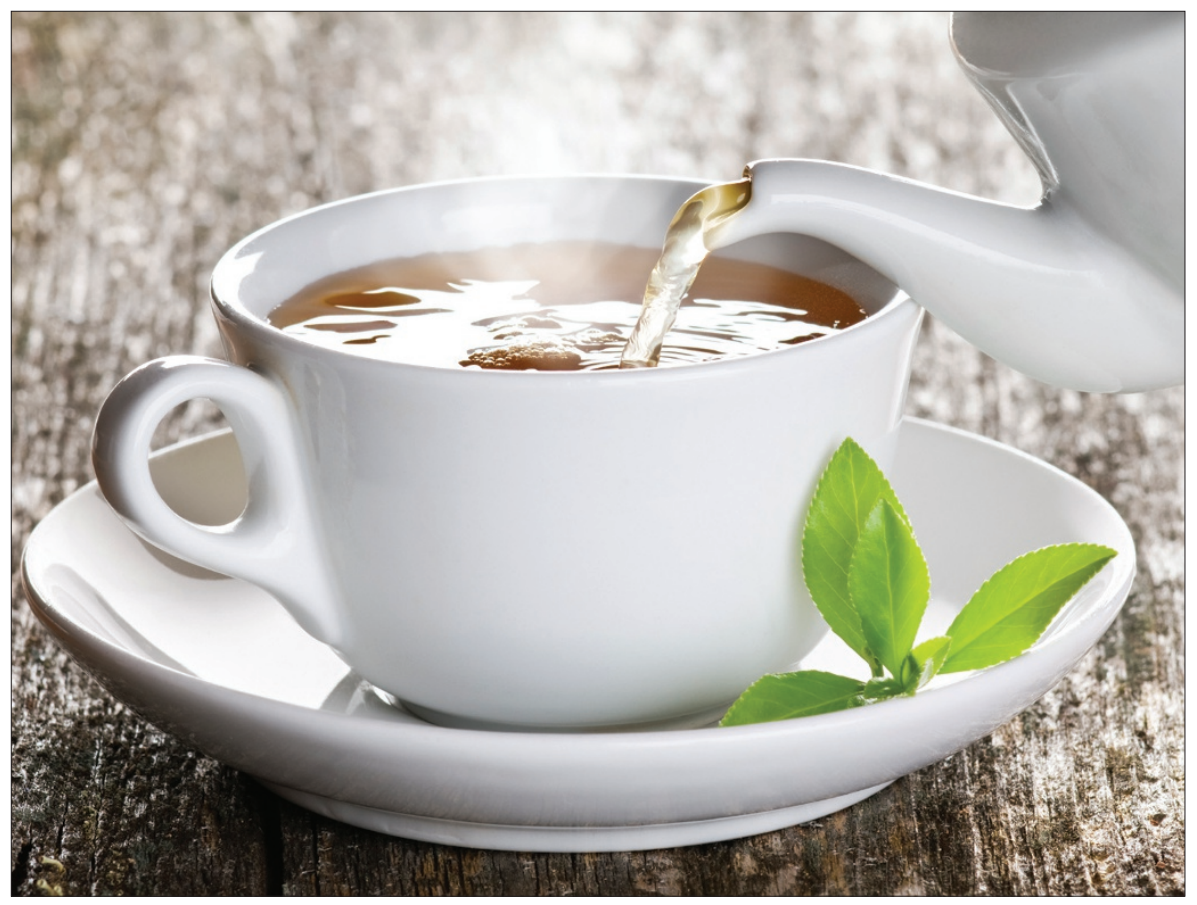

Japan. 'And since many of us enjoy green tea on a regular basis, my colleagues and I were eager to investigate the impact of green tea consumption on periodontal health, especially considering the escalating emphasis on the connection between periodontal health and overall health.'

Male participants aged 49-59 were examined on three indicators of periodontal disease: periodontal pocket depth (PD), clinical attachment loss (CAL) of gum tissue and bleeding on probing (BOP) of the gum tissue.

Researchers observed that for every one cup of green tea consumed per day, there was a decrease in all three indicators, therefore signifying a lower instance of periodontal disease in those subjects who regularly drank green tea.

Researchers think green tea's ability to help reduce symptoms of periodontal disease may be due to the presence of the antioxidant catechin. Previous research has demonstrated antioxidants' ability to reduce inflammation in the body, and the indicators of periodontal disease measured in this study, PD, CAL and BOP, suggest the existence of an inflammatory response to periodontal bacteria in the mouth.

By interfering with the body's inflammatory response to periodontal bacteria, green tea may actually help promote periodontal health, and ward off further disease.

\title{
BONE GROWTH AFTER TOOTH EXTRACTIONS EXAMINED
}

The use of platelet rich plasma (PRP) following tooth removal appears to speed healing and bone formation, according to a new study.

When a tooth is removed, poor healing can lead to excessive bone loss in the jaw that can delay tooth replacement, require costly reconstructive surgery, or even be impossible to fix. In the study, radiography techniques were used to detect bone changes after surgery to remove molars - specifically, the bilateral mandibular third molar. For each patient, one extraction site was treated with PRP and the site on the other side of the mouth was not, serving as the control. Three patients received $P R P$ on the right side and three on the left.

The patients returned after the operation for evaluations and digital radiographs at three days plus weeks 1,2 , $3,4,6,8,12,16,20$, and 24. Observers checked them visually to evaluate the extraction sites' tissue opening, bleeding, inflammation, facial oedema, and pain. The early radiographs found a significant increase in bone density in the PRP-treated sites.

According to the authors, the PRP treatment had a positive effect on bone density immediately following tooth extraction, while the control site had a decrease in bone density dur- ing the first week after surgery. After the initial two weeks, both sites had relatively parallel increases in bone density. Patients did not report significant differences in their perception of pain, bleeding, numbness, facial oedema or temperature between the different sites.

The article's authors suggest that faster bone formation could benefit patients who need immediate prostheses or dental implants, because the current four to six month wait for these could be reduced to two to four months if PRP is used. 'Platelet rich plasma to facilitate wound healing following tooth extraction appeared in the Journal of Oral Implantology (2010; 36: 11-23). 